\title{
A Case of an Atypical, Community Acquired Pneumonia: A CaSe Summary and TOPIC ReVIEW
}

\author{
David P. Cork MS IV, Joanne Kim MD, Jie Cui, MD
}

\section{Case Report}

A 59 year old gentleman with a past medical history of hypertension, hyperlipidemia, anxiety, depression, arthritis, and hypothyroidism presented with a five day history of fever to $102^{\circ} \mathrm{F}$. He complained of a three-day history of nausea, vomiting, and non-bloody, loose diarrhea, all of which had been persistent and worsening over the past week. Over the past two days, the patient had felt short of breath, and presented in a state of severe dyspnea. He had experienced an indolent course of illness, but was now concerned with his tachynpea and wheezing, and he had developed a productive cough with a small amount of yellow-brown sputum. He denied any hemoptysis as well as recent travel or sick contacts. On admission, the patient was taking hydrochlorothiazide, lisinopril, atorvastatin, levothyroxine and fluoxetine. He was also using over-thecounter ibuprofen for arthritic pain.

The patient was a smoker with a ten pack-year history. He denied intravenous drug use and occasionally drank alcohol. Family history was significant for paternal obstructive lung disease and coronary artery disease. On review of systems, the patient denied having a sore throat or any nasal congestion.

Vital signs upon presentation were temperature $101.0^{\circ} \mathrm{F}$, pulse 110 beats per minute, respiratory rate 40 breaths per minute, blood pressure $125 / 68 \mathrm{mmHg}$, and pulse oximetry of $86 \%$ on a non-rebreather mask. Upon physical examination, the patient was in moderate respiratory distress and unable to talk in complete sentences. He appeared mildly confused, but was awake, alert, and oriented. Jugular venous distention was not recognized. His cardiovascular exam was significant for tachycardia, but was without murmurs, rubs, or gallops. His pulmonary exam demonstrated coarse breath sounds bilaterally with diffuse, rhonchi throughout all lung fields. His abdomen was soft, mildly distended with moderate obesity, and was without hepato-splenomegaly. He had no lower extremity edema and his musculoskeletal exam was grossly normal. His skin was warm and moist, with good capillary refill $(<2 \mathrm{sec})$.

Laboratory studies upon admission demonstrated electrolyte abnormalities, including a sodium of $125 \mathrm{mmol} / \mathrm{L}$, chloride of $91 \mathrm{mmol} / \mathrm{L}$, blood urea nitrogen of $83 \mathrm{mmol} / \mathrm{L}$ and creatinine of $5.6 \mathrm{mmol} / \mathrm{L}$. His complete blood count was within normal limits. Blood gas demonstrated a $\mathrm{pH}$ of 7.22 carbon dioxide pressure of $45 \mathrm{mmHg}$, and oxygen pressure of $69 \mathrm{mmHg}$. Cardiac enzymes were negative and brain natriuretic peptide was $43.9 \mathrm{ng} / \mathrm{L}$. His coagulation studies were within normal limits, as was his thyroid stimulating hormone. A urinalysis showed a slightly cloudy specimen with mild proteinuria. Clostridium difficile antigen was negative.
Figure 1. Imaging upon admission

Chest $x$-ray - A bilateral infiltrative process is seen consuming the majority of the right lung.
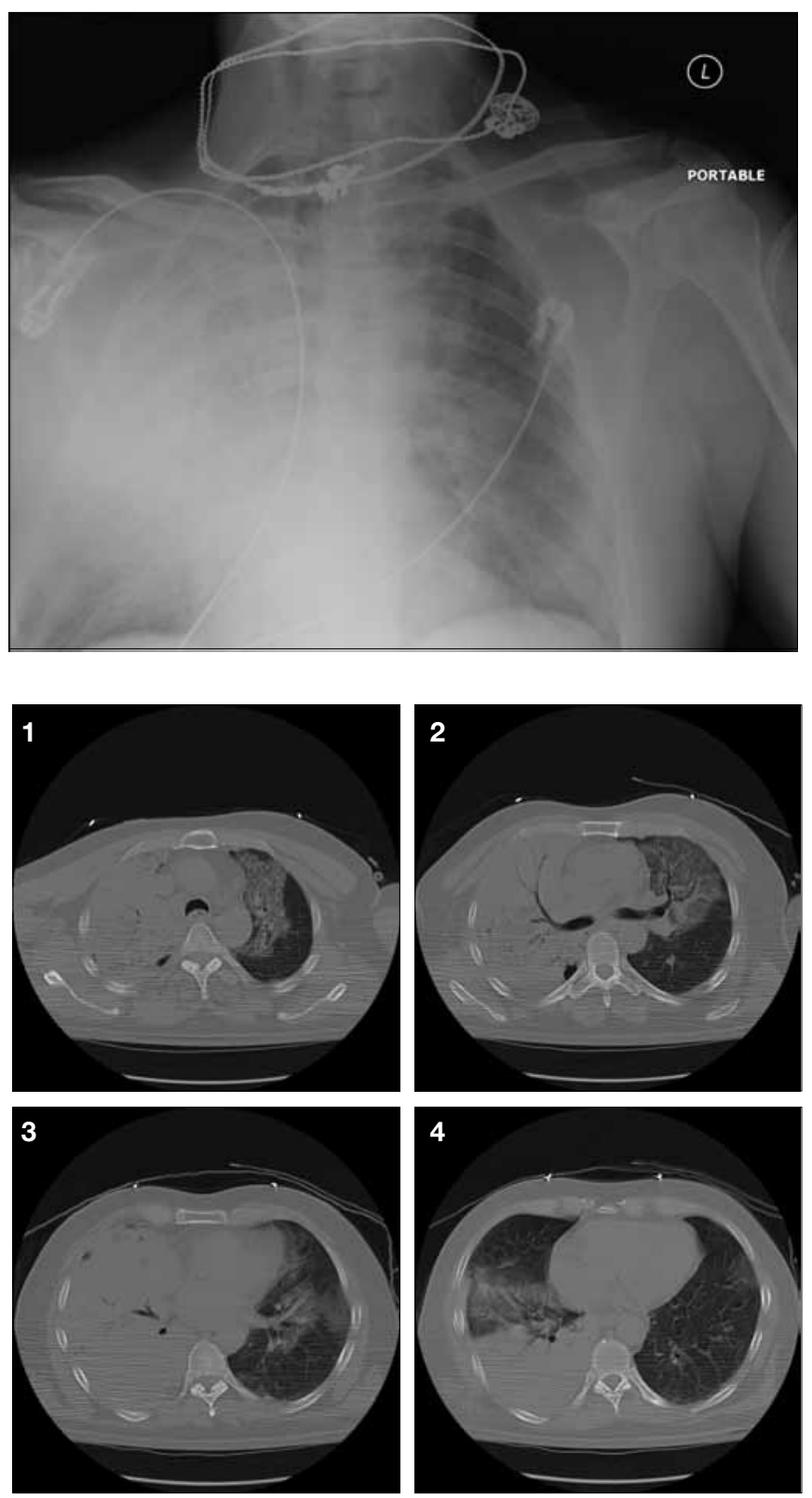

Figure 2. CT Chest without contrast - As the images move superiorly to inferiorly, note the dense consolidation of the right upper and lower lobes, as well as the groundglass appearance to the infiltrative process of the left upper lobe. 
The patient was intubated in the Emergency Department due to severe dyspnea, hypoxia, and respiratory failure. Blood, urine and stool cultures were obtained. The patient was started on empiric antibiotic coverage for signs of possible systemic inflammatory response system with consolidative pneumonia with pipercillin-tazobactam, vancomycin, and azithromycin. Given the patient's history of diarrhea, dyspnea, mild confusion, and hyponatremia, a Legionella urine antigen was also sent. The patient was admitted to the medical Intensive Care Unit, and nephrology and infectious disease services were consulted.

The patient's urine Legionella antibody assay returned positive, and antibiotic coverage was then narrowed to azithromycin. Treatment was planned for 21 days. After nine days of ventilatory support, the patient was weaned and extubated without complication. Upon questioning, the patient explained that he had recently been working to remodel his bathroom, including removing a finished bath tub which was designed to lay a top the previous tub. The oldest tub was full of stagnate water which had been pooled there for approximately six months before this planned bathroom remodel. This added a potential source of Legionella infection to the overall clinical story.

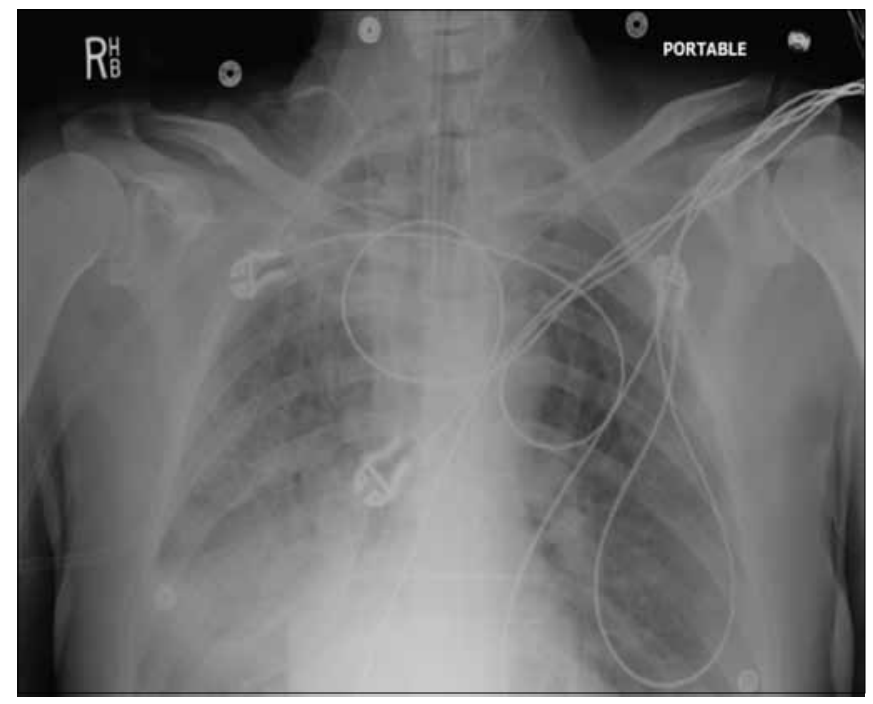

Figure 3. CXR upon extubation - There has been interval clearance of the infiltrative process of the right lung. Residual patchy infiltrates remain in the left lung base and right middle and lower lobes.

\section{Introduction to Legionella and Legionnaire's Disease Background}

First identified in Philadelphia in 1976, Legionella has become recognized as a common atypical pathogen. Legionella is ubiquitous and classified as a fastidious gram-negative coccobacilli, representing a genus with over fifty subgroups. L. pneumophila comprises at least sixteen different subgroups, many of which require very specific culture growth media. Legionellosis refers to two clinical syndromes caused by the genus Legionella: 1) Legionnaire's disease, a syndrome of pneumonia, and 2) Pontiac fever, an acute febrile illness that tends to be self-limited in nature.

\section{Epidemiology}

Legionella is noted as one of the top four causes of community acquired pneumonia (CAP) amongst the immunocompromised population, and is a common culprit of hospital-associated pneumonia. In fact, the incidence of Legionella as a cause of sporadic community-acquired pneumonia ranges from $2-15 \%$ of all cases requiring admission to a hospital.

\section{Transmission of Disease}

Transmission of Legionella is accomplished by way of aerosol inhalation or by micro-aspiration of water contaminated with the organism. ${ }^{1}$ Cooling towers, ultrasonic mist machines such as those used by grocery stores, respiratory equipment, and whirlpool baths have been commonly cited as vectors for disease transmission. Water distribution systems in nursing homes, workplaces, and private residences have each been implicated as primary sources of Legionellosis. Indeed, potable water sources have been associated with numerous reports of Legionella infection. The species L. pneumophila is capable of infecting and replicating in various protozoa found in soil and water, and may have increased virulence if replication occurs within amoeba. ${ }^{1}$ Due to L. pneumophila's virulence, Legionella pneumonia is recognized as manifesting a more severe pneumonia than other bacteria commonly associated with CAP.

The group of atypical CAP represents systemic infectious diseases that can primarily or secondarily infect the lungs. Atypical CAP differs from CAP in the way the specific bacteria infect host cells. Atypical bacteria are facultative intracellular organisms most susceptible to tetracylines, macrolides, or quinolones. Indeed, Legionella species are capable of exponential multiplication within human monocytes and alveolar macrophages. An intact cellular immune response is thus necessary to inhibit intracellular replication, with use of activated macrophage and monocyte cell-mediated immunity. Patients with immunesuppression have neutrophil ingestion of the organisms after complement and antibody have targeted the organism, but often are unable to effectively kill the bacteria. ${ }^{2}$ Typical pneumonias more commonly are comprised of organisms that invade interstitial spaces between cells and trigger neutrophil activation with cytokine release, thereby activating a generalized and immediate immune response.

\section{Risk Factors and Disease Manifestations}

Underlying disease is a major risk factor for acquisition of this disease. The risk factors most commonly implicated are cigarette smoking, chronic lung disease, and immunosuppression. Severely immunocompromised patients are said to fare worse in terms of disease severity, which may include lung abscess and bacteremia. They are also at risk for extra-pulmonary infections including sinusitis, pancreatitis, peritonitis, pyelonephritis, and most commonly, cardiac manifestations such as myocarditis, 
Table 1. Common Clinical Features of Legionnaire's Disease

\begin{tabular}{|l|l|l|}
\hline Organ Involved & Common Features & Argues Against Legionnaires' Disease \\
\hline Central Nervous System & Confusion & Meningeal Signs \\
\hline Upper Respiratory Tract & None & Sore throat, ear pain, bullous myringitis, otitis media \\
\hline Cardiac & Relative bradycardia & Splenomegaly \\
\hline Gastrointestinal & Loose, watery stools & $\begin{array}{l}\text { Hepatic tenderness, RLQ pain, LUQ tenderness, } \\
\text { peritoneal signs }\end{array}$ \\
\hline Renal & Renal insufficiency & CVA tenderness, chronic renal failure \\
\hline
\end{tabular}

pericarditis, postcardiotomy syndrome, and endocarditis (Table 1). ${ }^{3}$ Nasogastric tubes have been implicated as sources of nosocomial legionellosis as well. ${ }^{1}$ Also, patients who have recently undergone head and neck surgery, and therefore are susceptible to aspiration, have been noted as an at-risk population. ${ }^{1}$ Common clinical features of community-acquired pneumonias include: cough, fever, pleuritic chest pain, sputum production, and dyspnea. Scant sputum production is more typical of atypical community-acquired pneumonias, whereas typical pneumonias contracted within the community are more commonly characterized by mucopurulent sputum production.

\section{Differential Diagnosis}

- Chlamydia pneumonia

- Mycoplasma pneumonia

- Fungal pneumonia

- Viral pneumonia

- Q Fever

- Psittacosis

Owing to its potential degree of severity and its resistance to beta-lactam antibiotic therapy, Legionella represents the most important nonzoonotic CAP pathogen to differentiate from CAP pathogens. 2 With observation of commonalities amongst patients infected with Legionella, it is possible to make presumptive diagnoses based on clinical presentation. Therefore, it is important to keep in mind the following differentiating features frequently seen with Legionella infection when narrowing a differential diagnosis:

a. relative bradycardia in the context of a febrile illness 1. limits diagnosis to Legionella, Q fever, and psittacosis

b. unexplained mental confusion, including cerebellar ataxia

c. loose, watery stools

\section{Clinical Diagnosis}

Pneumonia is the predominant clinical syndrome. The disease may represent a broad spectrum of illnesses though, ranging from mild cough to respiratory failure. Typically seen early in the disease course are non-specific symptoms and signs such as fever, malaise, myalgias, headache, and anorexia. ${ }^{1}$ Cough tends to be only slightly productive, and temperature commonly exceeds $104^{\circ} \mathrm{F}$. Diarrhea may be seen in approximately $25 \%$ of cases, with complaints of watery rather than bloody stools; ${ }^{3}$ loose, watery diarrhea was reported in approximately $6 \%$ of other types of community-acquired pneumonias. ${ }^{3}$ Other common characteristic findings are relative bradycardia, which is most common among the elderly or those with advanced disease states and hyponatremia (serum sodium levels less than 130 $\mathrm{mg} / \mathrm{dL}$ ). Additionally, in patients demonstrating an increased cold agglutinin titer, which is commonly checked for suspected mycosplasma infections, the diagnosis of Legionella pneumonia is essentially ruled out. Due to the variance in severity of disease, as well as specialized lab tests required to confirm the diagnosis, Legionellosis is often underdiagnosed. ${ }^{5}$

The most important test for Legionnaire's disease is the isolation of the organism by culture on buffered charcoal yeast extract. Obtaining adequate sputum for culture can often be problematic in these patients however. When Legionnaire's is considered or suspected, a urine antigen test, Legionella culture, and a direct fluorescent antibody (DFA) staining from sputum or tissues should be considered. Indirect immunofluorescence assay (IFA) and/or enzyme-linked immunosorbent assay (ELISA) are also possible, but are not as useful in clinical decision making, as increased titers over four to eight weeks are required for confirmatory diagnoses (a fourfold rise in the titer of serum IgG antibody to L. pneumophila with final titer of at least $1 / 128$ is commonly considered positive). ${ }^{6}$ The urine antigen test is an enzyme immunoassay with sensitivity of approximately $70 \%$, and nearly $100 \%$ sensitivity; ${ }^{7}$ this test however applies to L. pneumophila serotype 1, which represents only $80-90 \%$ of Legionella infections. ${ }^{7}$ In contrast, the DFA test (sensitivity $33-68 \%$, specificity $99-100 \%$ ) can be performed in a manner of a few hours. ${ }^{8}$ ELISA tests are generally preferred over IFA tests nowadays, due to reportedly improved sensitivities (80\% and $70 \%$, respectively). ${ }^{8,9}$ Unfortunately this type of serum testing also lacks sensitivity for detecting serotypes other than serotype 1 , as with the urine tests.

\section{Treatment}

Quinolones or doxycyline remain first-line therapy. Delay in initiation of treatment significantly increases mortality. ${ }^{1}$ 
Table 2. Non-Specific Laboratory Tests

\begin{tabular}{|c|c|c|}
\hline Nonspecific Laboratory Tests & Common Features & Argues Against Legionaire's Disease \\
\hline Gram stain (sputum) & Few mononuclear cells, few/no bacteria & Purulent sputum, single predominant organism \\
\hline White Blood Cell Count & Leukocytosis, relative lymphopenia & Leucopenia, thrombocytosis, thrombocytopenia \\
\hline Pleural Fluid & Exudative & Red blood cells, decreased $\mathrm{pH}$, decreased glucose \\
\hline Aminotransferases & Mildly elevated (2-5x normal) & Markedly elevated (10x normal) \\
\hline Serum Phosphorus & Decreased (early) & Normal value does not rule out Legionnaire's Disease \\
\hline Creatine Phosphokinase & Increased (early) & Normal value does not rule out Legionnaire's Disease \\
\hline C-Reactive Protein & Increased (early) & Normal value does not rule out Legionnaire's Disease \\
\hline Ferritin & Highly elevated (>2x normal) & $\begin{array}{l}\text { Normal/slightly increased early. Normal value does not rule } \\
\text { out Legionnaire's Disease }\end{array}$ \\
\hline Cerebral Spinal Fluid & No pleocytes & Red blood cells, decreased glucose, decreased lactate \\
\hline Urinalysis & Microscopic hematuria & Gross hematuria, pyuria, hemoglobinuria \\
\hline Stool & Watery stools & Blood or mucus \\
\hline
\end{tabular}

Treatment duration is two weeks with quinolones, or two to four weeks with other antibiotics. ${ }^{1}$ Another treatment option is macrolides, however there is variability between drug efficacy in this class for eradicating Legionella. For instance, erythromycin has been associated with therapy failures. ${ }^{1}$ Other macrolides, such as azithromycin are said to have better in vitro activity and improved pulmonary tissue penetration. ${ }^{1}$ Overall, quinolones have improved in vitro activity and intracellular penetration compared to macrolides. In patients who are severely ill, rifampin is recommended as an adjunct in combination therapy with a macrolide or quinolone, as it has high levels of activity against Legionella. Imipenem, trimethoprim-sulfamethoxazole, and clindamycin have all been proven efficacious for the treatment of Legionella pneumonia. ${ }^{9}$ Treatment of the underlying pneumonia will improve the associated diarrhea, electrolyte abnormalities, and other associated syndromes seen with Legionnaire's Disease.

\section{Prognosis}

Patients with Legionnaire's Disease usually experience symptomatic improvement within three to five days if treated early with an effective antibiotic. With early treatment and appropriate drug choice, the mortality rate for immunocompromised patients approaches that of immuno-competent patients. ${ }^{10}$ However, mortality can vary widely and is noted to be as high as $80 \%$ in cases, particularly in patients with underlying disease where lack of prompt recognition and treatment initiation exist, and in nosocomial or large outbreaks. Decreasing mortality trends have been noted recently due to increased awareness and consideration of the disease as a possible infectious etiology.

\section{References}

1. Stout J, Yu V. Review Article of Legionellosis. New England Journal of Medicine. September 4, 1997. Volume 337, Number 10.

2. Jacobson K. Legionella Pneumonia in Cancer Patients. Medicine. Volume 87, Number 3, May 2008.

3. Sopena N. Comparative Study of the Clinical Presentation of Legionella Pneumonia and Other Community-Acquired Pneumonias. Chest. May 1998, pp 1195-1200.

4. Cunha B. Atypical pneumonias: current clinical concepts focusing on Legionnaire's disease. Current Opinion in Pulmonary Medicine 2008, 14:183-194.

5. Sabria M. Hospital-acquired legionellosis: solutions for a preventable infection. The Lancet. Infectious Diseases, Vol 2. June 2002.

6. Gacouin A. Severe pneumonia due to Legionella pneumophila: prognostic factors, impact of delayed appropriate antimicrobial therapy. Intensive Care Medicine. 2002. 28:686-691.

7. Mandell LA, Wunderink RG, Anzueto A, et al.; Infectious Diseases Society of America/American Thoracic Society consensus guidelines on the management of community-acquired pneumonia in adults.; Clin Infect Dis; 2007; Vol. 44 Suppl 2 pp. S27-72.

8. Waterer G. Legionella and community-acquired pneumonia: a review of current diagnostic tests from a clinician's viewpoint. American Journal of Medicine. Volume 110, Issue 1, January 2001. pp 41-48.

9. Diederen BMW. Legionella spp. And Legionnaire's Disease. Review. Journal of Infection. 2008. 56, 1-12.

10. Edelstein PH. Antimicrobial chemotherapy for legionnaire's disease: a review. Clinical Infectious Diseases 1995;21:Suppl 3:5265-76. 